\title{
Numerical simulation of atmospheric Lamb waves generated by the 2022 Hunga-Tonga volcanic eruption
}

\author{
Angel Amores ${ }^{1}$, Sebastian Monserrat ${ }^{2}$, Marta Marcos ${ }^{1,2}$, Daniel Argüeso ${ }^{2}$, \\ Joan Villalonga ${ }^{3}$, Gabriel Jordà ${ }^{3}$ and Damià Gomis ${ }^{1,2}$. \\ ${ }^{1}$ Instituto Mediterráneo de Estudios Avanzados (UIB-CSIC), Esporles, Spain. \\ 2 Departament de Física (UIB), Palma, Spain \\ ${ }^{3}$ Centre Oceanogràfic de Balears, Instituto Español de Oceanografía (IEO-CSIC), Palma, Spain
}

This manuscript is a non-peer reviewed preprint submitted to EarthArXiv that has been submitted to Geophysical Research Letters.

\section{Key Points:}

- The underwater Hunga-Tonga volcano exploded generating Lamb waves that traveled around the Earth several times.

- We simulate these waves using a hydrostatic shallow water equation oceanic model.

- The results closely follow the observations of atmospheric pressure perturbations.

Corresponding author: Angel Amores, angel.amores@uib.es 


\begin{abstract}
On January $15^{t h}, 2022$, at 4:30 UTC the eruption of the Hunga-Tonga volcano, in the South Pacific Ocean, generated a violent underwater explosion. In addition to tsunami waves that affected the Pacific coasts, the eruption created atmospheric pressure disturbances that spread out in the form of Lamb waves. The associated atmospheric pressure oscillations were detected in high-frequency in-situ observations all over the globe. Here we take advantage of the similarities in the propagation and characteristics between atmospheric Lamb waves and long ocean waves and we use a 2DH ocean numerical model to simulate the phenomenon. We compare the outputs of the numerical simulation with in-situ atmospheric pressure records and with remote satellite observations. The signal in the model matches the observed atmospheric pressure perturbations and reveals an excellent agreement in the wave arrival time between model and observations at hundreds of locations at different distances from the origin.
\end{abstract}

\title{
Plain Language Summary
}

The underwater explosion of the Hunga-Tonga volcano in the South Pacific Ocean generated atmospheric pressure disturbances, known as Lamb waves, that propagated and surrounded the globe several times. In this study, we exploit the similarities between atmospheric Lamb waves and long waves in the ocean (e.g., tsunamis) to simulate their propagation using an ocean numerical model. The comparison of our results with remote satellite data and in-situ atmospheric pressure records reveals that our model correctly reproduces the propagation of the atmospheric disturbances generated by the volcano explosion.

\section{Introduction}

On January $14^{\text {th }}, 2022$ the underwater Hunga-Tonga volcano, located in the South Pacific Ocean, erupted in a one-in-a-thousand year event (Klein, 2022). The volcano, located between the uninhabited islands of Hunga Tonga and Hunga Ha'apai of the Kingdom of Tonga, is part of the Tonga-Kermadec Islands volcanic arc and has been active since its first historical eruption in 1912 (Global Volcanism Program, 2022). The volcano had emerged after an eruption that started in December 2014. This recent eruption resulted in material being deposited and merged with the Hunga Ha'pai island, creating an area of around $2 \mathrm{~km}$ of diameter and maximum height of $120 \mathrm{~m}$ above sea level (Cronin et al., 2017). According to the Global Volcanism Program (2022), the strongest eruption began on January $15^{\text {th }}$ at 17:30 local time (4:30 UTC) with a plume reaching $30 \mathrm{~km}$ in the atmosphere and $600 \mathrm{~km}$ in diameter, making it visible by multiple satellite observations. Observations of Sentinel-2 satellites revealed massive changes in the surface area and the disappearance of the formerly deposited volcanic material. The explosive eruption, whose power has been estimated to be equivalent to somewhere between 4 to 18 megatons of TNT (https://earthobservatory.nasa.gov/images/149367/dramatic -changes-at-hunga-tonga-hunga-haapai), generated tsunami waves (warnings were issued across several countries in the Pacific coasts) and also atmospheric shock waves that propagated across the globe and were detected by the NASA Aqua satellite as concentric wave patterns (Adam, 2022).

Such amount of energy liberated into the atmosphere by the violent eruption is expected to generate various types of atmospheric waves with different spectral energy content, including inertia gravity waves, infrasound waves or Rossby waves, making the atmospheric wave pattern close to the source very intricate. Among these atmospheric perturbations, the type of wave which is expected to optimally transfer energy over long distances, and therefore the one expected to dominate far away from the source, is the Lamb wave mode, which was first introduced by Horace Lamb (Lamb, 1881). This has been 
observed in earlier similar events, as for example the well-known Krakatoa volcanic eruption in 1883 (Symons, G. J. (ed.), 1888).

Lamb waves are non-dispersive atmospheric waves, whose energy is optimally transmitted far away from the source with minor losses. They arise as solutions of the momentum equations with zero vertical velocity, meaning that Lamb waves have purely horizontal motion, occupying the full depth of the troposphere and with a maximum pressure signal at the surface. These waves are only slightly affected by the Earth's rotation and travel at the speed of sound in the media (Gossard \& Hooke, 1975). Assuming an isothermal troposphere, the phase velocity of the Lamb waves, $C_{T}$, is only affected by the air temperature and is defined as:

$$
C_{T}=\sqrt{\frac{\gamma \cdot R \cdot T}{M}}
$$

where $\gamma=1.4$ is the ratio of specific heat of air corresponding to the range of atmospheric temperatures, $R=8314.36 \mathrm{~J} \cdot \mathrm{kmol}^{-1} \cdot K^{-1}$ is the universal gas constant, $M=28.966 \mathrm{~kg} \cdot \mathrm{kmol}^{-1}$ is the molecular mass for dry air and $T$ is the absolute temperature.

Due to their particular characteristics, the propagation of Lamb waves through the atmosphere with spatially varying temperature is analog to the behavior of oceanic long waves propagating over an ocean with variable depth. Long waves in the ocean are also non-dispersive barotropic waves traveling with a phase velocity, $C_{H}$, given by

$$
C_{H}=\sqrt{g \cdot H}
$$

where $g=9.81 \mathrm{~m} \cdot \mathrm{s}^{-2}$ is the gravity acceleration and $H$ is the ocean depth.

Long waves in the ocean have been successfully simulated using $2 \mathrm{DH}$ shallow water equation models, as for example, the propagation of tsunami waves and their arrival times at remote coastal locations (e.g. Titov et al. (2005)).

Given these similarities between atmospheric Lamb waves and oceanic shallow water waves, we propose to simulate the atmospheric Lamb wave generated after the HungaTonga volcano explosion using a vertically-integrated hydrodynamic ocean model. To do so, a simple relationship between the vertically integrated atmospheric temperature and the equivalent ocean depth is obtained from eq. 1 and 2

$$
H=\frac{\gamma \cdot R \cdot T}{M \cdot g}
$$

This study is organized as follows: in section 2 the data and the model used for the simulations as well as the way it was initialized are described. Results of the simulations are compared with remote and in-situ observations in section 3 and a summary and conclusions are presented in section 4 .

\section{Data and Methods}

The numerical ocean hydrodynamic model SCHISM (Semi-implicit Cross-scale Hydroscience Integrated System Model, V5.9.0; Y. J. Zhang et al. (2016)) was used to simulate the atmospheric Lamb waves generated by the volcano explosion. We have used its dynamic core, which is a derivative product built from the original SELFE (v3.1dc; Y. Zhang and Baptista (2008)), in 2DH barotropic mode. It solves the vertically-integrated hydrostatic Navier-Stokes equations with shallow water approximation. The model domain covers the entire globe with an unstructured triangular computational grid of $0.25^{\circ}$ resolution with 1036800 nodes and 2070720 elements. The simulation starts on January 
$15^{\text {th }} 2022$ at 04:30 UTC coinciding with the volcano explosion and has a duration of 5 days. The computational time step was set to $1 \mathrm{~min}$ and the variables were saved every $5 \mathrm{~min}$ at each computational grid point.

To define the equivalent water depth in the model (see equation 3), we used the atmospheric temperature fields obtained from ERA5 reanalysis (https://cds.climate .copernicus.eu/). ERA5 is a comprehensive reanalysis that spans from 1979 to nearreal time and integrates historical observations into global estimates using advanced modeling and data assimilation systems. ERA5 data is provided at 1-hour temporal resolution and $0.25^{\circ}$ spatial resolution. A time-varying temperature field over the domain was defined to represent the vertically-averaged atmospheric temperature. For the results shown, the simplest approach was taken. The temperature field has been computed as the average between the temperature at $2 \mathrm{~m}$ (obtained from ERA5 data on single levels; Hersbach et al. (2018b)) and the temperature at the top of the troposphere (whose altitude has been taken as constant at $100 \mathrm{hPa}$ level obtained from ERA5 data on pressure levels; Hersbach et al. (2018a)). The results do not vary significantly when more complex algorithm is used to define the temperature field. Tropospheric temperatures were translated into equivalent depth fields using eq. 3, which in turn were incorporated into the model through the bathymetry. As such, the bathymetry field was updated every hour to take into account air temperature variations estimated from ERA5 hourly data.

The initial perturbation created by the volcano eruption was simulated using an equivalent atmospheric pressure perturbation of $50 \mathrm{hPa}$. In the model, this was introduced as an instantaneous sea level perturbation at the start of the simulation, which had a cylinder-like shape of $60 \mathrm{~km}$ radius and $50 \mathrm{~cm}$ height. The intensity and the extend of the initial perturbation were chosen to match the amplitude and frequency of the available observations. Other shapes such as a Gaussian and semi-spherical perturbations were also tested for the initial forcing with similar results.

The outputs of the hydrodynamic model are provided as sea surface displacements. We apply the inverted barometer equivalence to convert the sea level response into an atmospheric pressure signal. This approach corresponds to a decrease of $1 \mathrm{hPa}$ for every $\mathrm{cm}$ of water elevation, and vice versa. The simulation took a total of $6 \mathrm{~h}$ to complete with $23 \mathrm{CPU}$.

The simulation was validated against in-situ surface atmospheric pressure records obtained from different sources (see the map in Fig. 2 to see the spatial distribution of the stations). A total of 889 station were retrieved from NOAA Automated Surface/Weather Observing Systems (ASOS/AWOS, downloaded from https://mesonet.agron.iastate .edu/request/asos/1min.phtml\#) spread across all United States, including Hawaii, Alaska and Puerto Rico. From these, only those with less than $10 \%$ of missing values were retained, which left a total of 714 stations ( $20 \%$ of them were removed). The time from these stations was described as being UTC. However, some of them showed a time difference with surrounding stations that matched the shift between UTC and local time, which suggests their time record was actually in local time units, thus they were corrected accordingly. Finally, other stations with clear anomalous behavior when compared with surrounding stations were removed. The total number of stations finally used was 660 . A time series of atmospheric pressure from Ciutadella (Balearic Islands, Spain) with a temporal resolution of 30 seconds was obtained from the Balearic Islands Coastal Observing and Forecasting System (SOCIB, available at https://www.socib.es/?seccion= observingFacilities\&facility=mooring). Another time series from Kadhdhoo (https:// mv.geoview.info/kadhdhoo,7909905), in the Maldives, with a 10 minutes temporal resolution was also used to compare with the model outputs. Atmospheric pressure records where also obtained from the Australian Bureau of Meteorology at three locations (Sydney Observatory Hill, Perth Airport and Darwin Airport) with 1 minute temporal resolution. Since the period of the generated Lamb wave was around 40 minutes, the at- 
mospheric pressure records were band-pass filtered with cut-off periods between 2 hours and 15 minutes.

The simulation was also qualitatively compared to satellite observations to further assess the realism of the wave propagation. Infrared data from the Geostationary Operational Environmental Satellite (GOES-R) program (obtained from https://www.ncdc .noaa.gov/airs-web/search) and the European Organisation for Exploitation of Meteorological Satellites (EUMETSAT; downloaded from https://navigator.eumetsat . int/product/EO:EUM:DAT:MSG:HRSEVIRI) were used at 15-min temporal resolution for the first 24 hours since the eruption. The Pacific region was represented by the GOES17 satellite with imagery from the IR10.3 channel with a spatial resolution of $5424 \times 5424$ pixels. The 0-degree region was observed by the Meteosat-11 satellite (High Rate SEVIRI Level 1.5 Image Data) with data from the IR10.8 channel with a spatial resolution of $3712 \times 3712$ pixels. For the sake of visualization of the atmospheric pressure wave footprint in the satellite IR observations we used, at each time step, their second timederivative. These fields were subsequently spatially filtered with a 50 (100) pixel window for GOES-17 (Meteosat-11) satellite observations with the filter described in Amores et al. (2018).

\section{Results}

A qualitative comparison of the model results with satellite observations during the first travel of the Lamb waves (from the origin to the antipodes in Northern Africa) reveals that the simulation closely follows the spatial pattern of the satellite measurements (Fig. 1). Note that we are comparing the observed and modeled spatial footprints of the waves, but using different variables. The relevant parameter here is thus the location of the wave rather than its amplitude. Panels $a-f$ show the propagation of the Lamb wave over the Pacific captured by GOES-17 satellite from 15 minutes after the explosion until January $15^{\text {th }} 10: 30$ UTC. The wave is clearly observed in satellite images that also display a close agreement with the observations. Panels $g$ - $j$ show the travel of the wave captured by Meteosat-11 satellite from 17:30 to 20:30 UTC. In this case, although still identified, the wave signal is surrounded by noisier data probably due to a larger cloud coverage and/or lower spatial resolution offered by this satellite in comparison with GOES17. The wave is observed at 17:30 and 18:30 and it is still visible at 19:30 and 20:30, coinciding again with the pattern of the simulation.

Fig. 2 shows the comparison of 10 high-frequency atmospheric pressure records (colors help matching dots in the map and time series in the lower panel) at different distances from the volcano (indicated with a red star in the map) between January $15^{\text {th }}$ 04:30 UTC until January 18 ${ }^{\text {th }}$ 02:40 UTC. In addition, the temporal evolution of the simulation is available in Movie S1 in the Supplementary Material. The modeled time series (in grey) were extracted from the closer grid point to each station. At all locations the numerical simulation matches very well the time of arrival of the Lamb wave. At each site, 4 different passes are observed, except in the Ciutadella station (dark red), the closest to the volcano's antipodes in our database. In this station only two passes occur because of the overlapping of the northern and southern waves (see Movie S1 for a better visualization). The model better captures the first wave pass, as shown by both the arrival time and the wave amplitude. Once the Lamb wave has traveled farther distances and has interfered with its own and the environment, the patterns become more complex. However, the model is still able to correctly capture the arrival time in most cases.

Using all available atmospheric pressure records, we have quantified the performance of the approach by comparing the time of arrival of the first Lamb wave. To do so, we have determined the time when the first atmospheric pressure maximum is found at insitu pressure records and in the model simulation. Fig. 3 represents the scatter plot of modeled vs. observed arrival times of the first wave. There is an excellent agreement be- 
tween model and observations at all sites, with a $R^{2}$ larger than 0.98 and a root mean square difference (RMSD) of around 10 minutes (we remark here that the temporal resolution of the simulation is 5 minutes).

\section{Summary and Conclusions}

After Hunga-Tonga volcano explosion on January $15^{\text {th }}$, 2022, atmospheric pressure records around the world measured high-frequency perturbations that traveled around the globe several times and that were consistent with the presence of atmospheric Lamb waves. We have numerically simulated the atmospheric Lamb waves generated by the volcanic eruption taking advantage of their similarities to ocean long waves. Namely, both types of waves propagate through the fluid as vertically integrated waves, with $2 \mathrm{D}$ horizontal motion and share the same dispersion relation. The analogy consists of defining an equivalent bathymetry in the ocean shallow water model that corresponds to the vertically averaged air temperature, which has furthermore temporal variability.

The results of the simulation mimic satellite and in-situ observations. In particular, when the outputs of the model are compared to atmospheric pressure records at different distances from the source, they display excellent matching in the arrival times of the perturbation. Therefore, the results confirm that the observed high-frequency surface pressure oscillations are the footprint of non-dispersive atmospheric Lamb waves originated by the eruption of the Hunga-Tonga volcano.

Despite being an idealized simulation, which neglects various factors that may affect different characteristics of the wave, the close agreement between the observation and the model suggests that the main physical mechanisms are well represented in our experiment. For example, our model does not consider the effect of orography. High mountain systems such as the Andes or Himalayas may cause reflections of the Lamb waves that are not represented in our simulation. We also made some assumptions in our approach, but they do not prevent us from correctly simulating the wave propagation. For example, we assumed the temperature to be constant in the vertical through the troposphere, but we found that using the average temperature was a good approximation to estimate the equivalent depth. We also assumed the air to be dry and thus, we considered that water vapor and humidity changes have only a minor effect on the propagation of the wave. In summary, we have shown how a vertically integrated hydrodynamic ocean model can be used to investigate and anticipate the propagation of atmospheric Lamb waves across an isotherm troposphere.

\section{Conflict of Interest}

The authors declare no conflicts of interest relevant to this study.

\section{Open Research}

Data Availability Statement: all the data used in this study can be accessed trough the links provided in the Data and Methods section.

The numerical simulation can be downloaded from: https://doi.org/10.5281/zenodo.5948860

\section{Acknowledgments}

This study was supported by the MOCCA project RTI2018-093941-B-C31 funded by MCIN/AEI /10.13039/501100011033/ and by FEDER Una manera de hacer Europa. It was also supported by grants PGC2018-099285-B-C21 and PGC2018-099285-B-C22 funded by MCIN/AEI/ $10.13039 / 501100011033$ and by "ERDF A way of making Europe" NextGenerationEU/PRTR. Angel Amores was funded by the Conselleria d'Educació, Universitat i Recerca del Gov- 
ern Balear through the Direcció General de Política Universitària i Recerca and by the Fondo Social Europeo for the period 2014-2020 (grant no. PD/011/2019). Daniel Argüeso was funded by Spanish Ministry of Science and Innovation through the EPICC Project (PID2019-105253RJ-I00) and the Beatriz Galindo Programme (BG20/00078). The authors are grateful to NOAA, EUMETSAT, the Australian Bureau of Meteorology and SOCIB to make their data freely available. Finally, we also thank to Dr. Ali Shareef for providing the atmospheric pressure data from the Maldives.

\section{References}

Adam, D. (2022). Tonga volcano eruption created puzzling ripples in earth's atmosphere. Nature. doi: 10.1038/d41586-022-00127-1.

Amores, A., Jordà, G., Arsouze, T., \& Le Sommer, J. (2018). Up to what extent can we characterize ocean eddies using present-day gridded altimetric products? Journal of Geophysical Research: Oceans, 123(10), 7220-7236. doi: https://doi.org/10.1029/2018JC014140

Cronin, S. J., Brenna, M., Smith, I., Barker, S., Tost, M., Ford, M., ... Vaiomounga, R. (2017). New volcanic island unveils explosive past. EOS, 98. doi: https://doi.org/10.1029/2017EO076589

Global Volcanism Program. (2022). Report on hunga tonga-hunga ha'apai (tonga). Bulletin of the Global Volcanism Network, Smithsonian Institution, 40:1. doi: https://doi.org/10.5479/si.GVP.BGVN201501-243040

Gossard, E., \& Hooke, W. (1975). Waves in the atmosphere. Amsterdam: Elsevier.

Hersbach, H., Bell, B., Berrisford, P., Biavati, G., Horányi, A., Muñoz Sabater, J., ... Thépaut, J.-N. (2018a). Era5 hourly data on pressure levels from 1979 to present. Copernicus Climate Change Service (C3S) Climate Data Store (CDS). doi: $10.24381 /$ cds.bd0915c6

Hersbach, H., Bell, B., Berrisford, P., Biavati, G., Horányi, A., Muñoz Sabater, J., ... Thépaut, J.-N. (2018b). Era5 hourly data on single levels from 1979 to present. Copernicus Climate Change Service (C3S) Climate Data Store (CDS). doi: $10.24381 /$ cds.adbb2d 47

Klein, A. (2022). Tongan volcano erupts. New Scientist, 253(3370), $7 . \quad$ Retrieved from https://www.sciencedirect.com/science/article/pii/ S0262407922000744 doi: https://doi.org/10.1016/S0262-4079(22)00074-4

Lamb, H. (1881). On the vibrations of an elastic sphere. Proceedings of the London Mathematical Society, s1-13(1), 189-212. Retrieved from https://londmathsoc.onlinelibrary.wiley.com/doi/abs/10.1112/plms/ s1-13.1.189 doi: https://doi.org/10.1112/plms/s1-13.1.189

Symons, G. J. (ed.). (1888). The eruption of krakatoa and subsequent phenomena. Report of the Krakatoa Committee of the Royal Society.

Titov, V., Rabinovich, A. B., Mofjeld, H. O., Thomson, R. E., \& González, F. I. (2005). The global reach of the 26 december 2004 sumatra tsunami. Science, 309(5743), 2045-2048. doi: 10.1126/science. 1114576

Zhang, Y., \& Baptista, A. M. (2008). Selfe: A semi-implicit eulerian-lagrangian finite-element model for cross-scale ocean circulation. Ocean Modelling, 21(3), 71-96. doi: https://doi.org/10.1016/j.ocemod.2007.11.005

Zhang, Y. J., Ye, F., Stanev, E. V., \& Grashorn, S. (2016). Seamless cross-scale modeling with schism. Ocean Modelling, 102, 64-81. doi: https://doi.org/10 $.1016 /$ j.ocemod.2016.05.002 

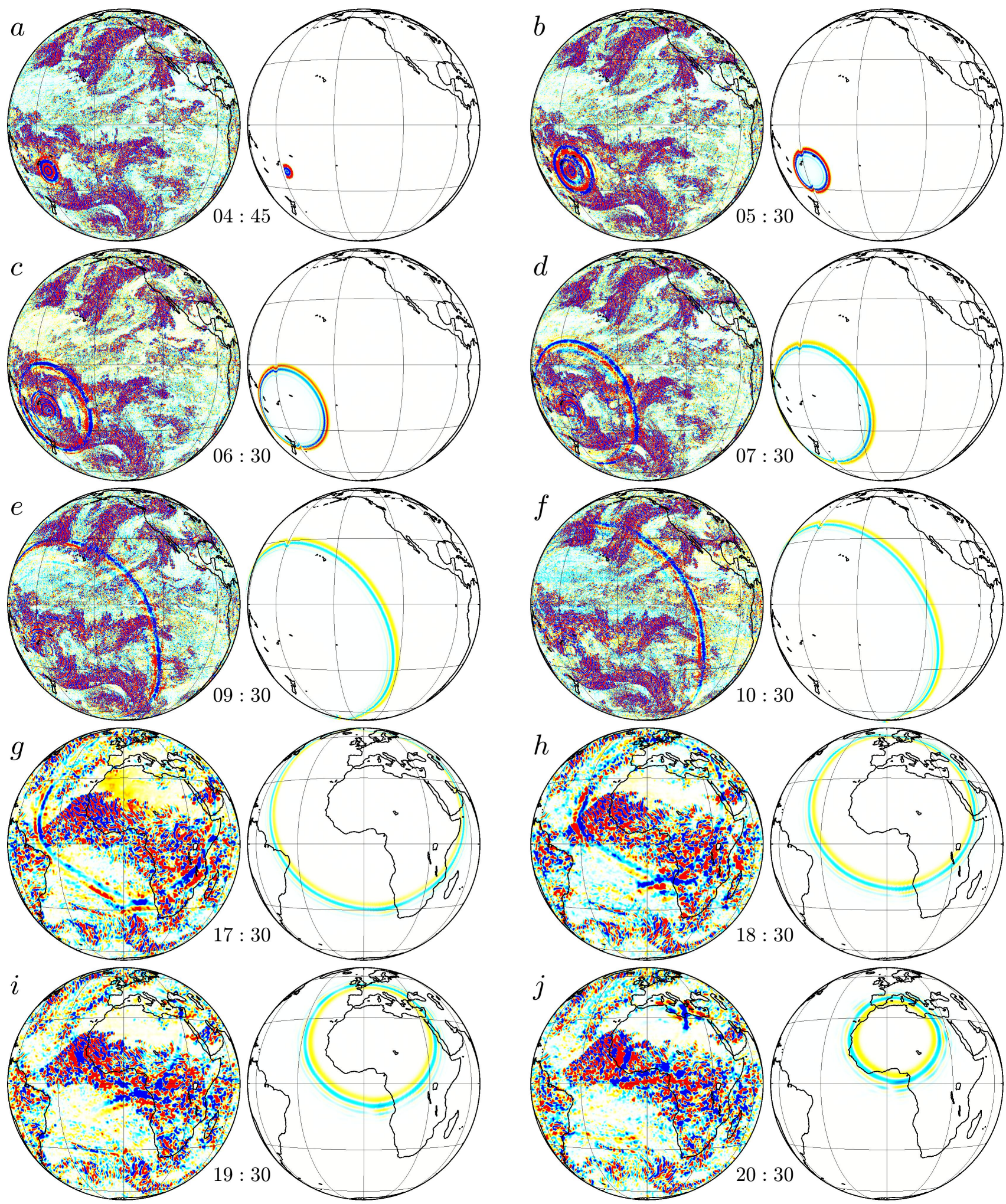

Figure 1. Comparison of the Lamb wave observed from satellite observations and the numerical simulation during January $15^{t h}$, 2020 at different times. Each panel shows the satellite observations at left and the corresponding simulation field at the right. Panels $a$ to $f$ correspond to observations from GOES-17 satellite while panels $g$ to $j$ correspond to observations from Meteosat-11 (see in the Data and Methods sections the details of the postprocessing performed). The colorscales are different for each satellite and numerical simulation and are fixed to provide a correct visualization. 

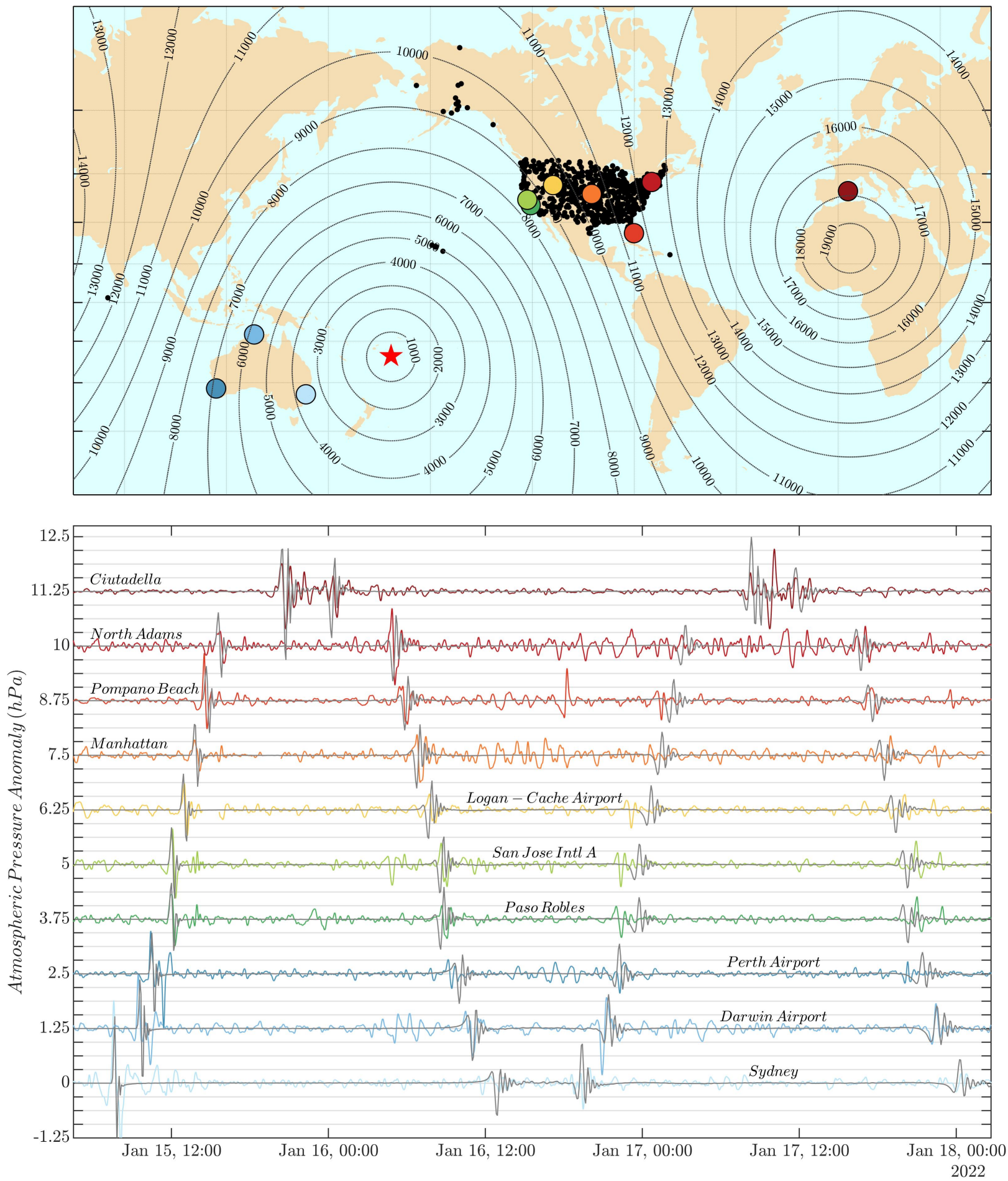

Figure 2. The upper panel shows the location from where of all atmospheric pressure records used were measured (black and colored dots). The red star indicated the location of the explosion. Contour lines indicate the distance from the location of the explosion in $\mathrm{km}$. The lower panel shows the comparison between 10 atmospheric pressure anomaly records (in different colors corresponding to the colored points from the upper panel) and the numerical simulation record at the closest grid point (black lines) from January $15^{\text {th }}$ 04:30 UTC until January $18^{\text {th }}$ 02:40 UTC. The different stations shown were selected to cover different distances from the origin of the Lamb wave. 


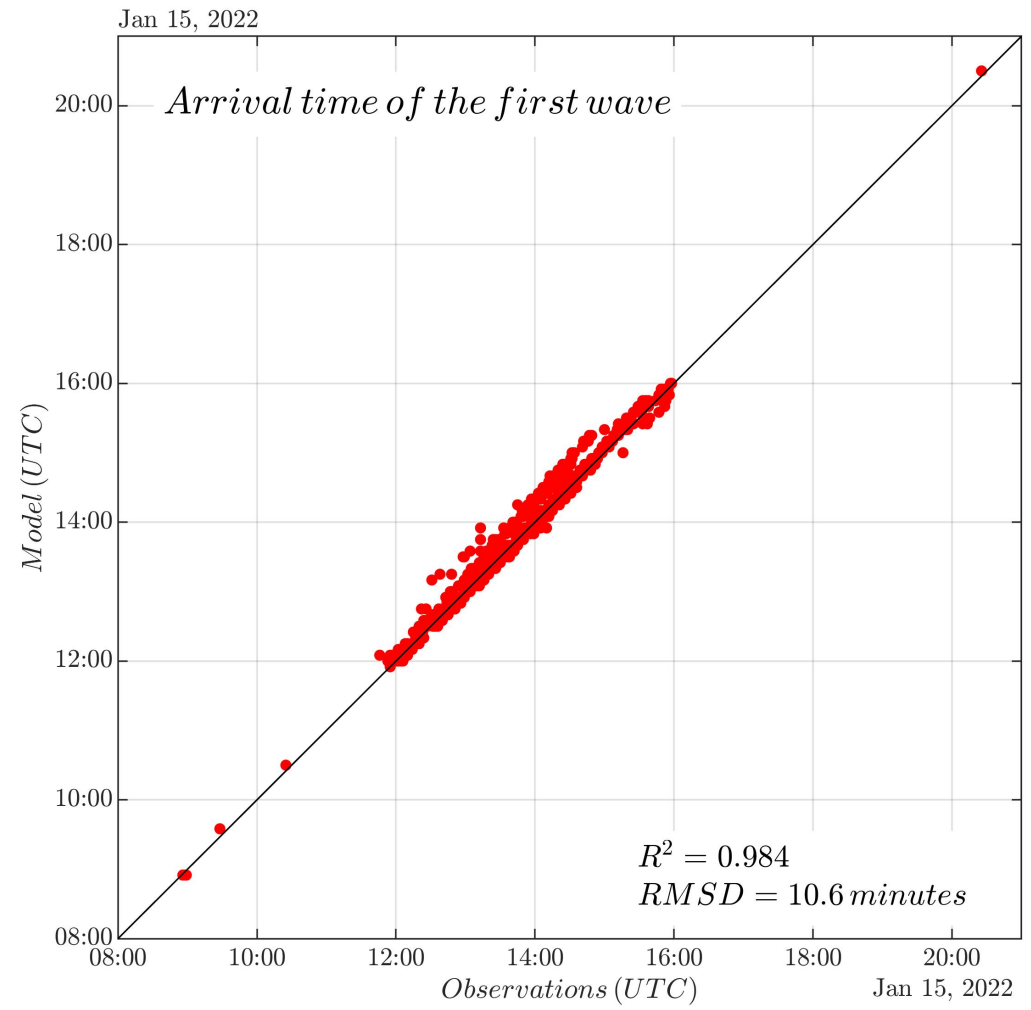

Figure 3. Comparison between the modeled arrival time of the first pass of the Lamb wave as a function of the observed arrival time. 